\title{
FATORES ASSOCIADOS À OCORRÊNCIA DE ANSIEDADE DOS ACADÊMICOS DE BIOMEDICINA
}

\section{Mayara Quadros Cardozo}

Discente de Biomedicina da Universidade do Extremo Sul de Santa Catarina (UNESC), Criciúma (SC), Brasil.

\section{Karin Martins Gomes}

Doutora em Ciências da Saúde pela Universidade do Extremo Sul de Santa Catarina (UNESC), Criciúma (SC), Brasil.

\section{Lee Gi Fan}

Fisioterapeuta; Mestre em Educação pela pela Universidade do Extremo Sul de Santa Catarina (UNESC), Criciúma (SC), Brasil.

\section{Maria Tereza Soratto}

Mestre em Educação; Docente no Curso de Biomedicina Universidade do Extremo Sul de Santa Catarina (UNESC), Criciúma (SC), Brasil.

\begin{abstract}
RESUMO: Estudo com objetivo de identificar os fatores associados à ocorrência de ansiedade nos acadêmicos de Biomedicina. Pesquisa quali-quantitativa, descritiva e exploratória. Estudo desenvolvido com 20 acadêmicos da $7^{\mathrm{a}}$ fase do curso de Biomedicina da Universidade do Extremo Sul Catarinense. Aplicou-se a Escala de Avaliação de Ansiedade de Hamilton e questionário semiestruturado sobre os fatores relacionados à ansiedade dos universitários em relação ao processo de ensino-aprendizagem, avaliação, comunicação profissional e relacionamento interpessoal, gerenciamento do tempo, formação profissional e ao trabalho de conclusão de curso. Os resultados da Escala apontaram nível de ansiedade de $65 \%$ grau leve; $25 \%$ grau moderado; $10 \%$ grau intenso dos acadêmicos de Biomedicina. Considera-se imprescindível o cuidado aos acadêmicos da Universidade, com foco no autocuidado e autoconhecimento. Sugere-se um aprofundamento da pesquisa, com maior número de acadêmicos, contemplando as diversas fases do curso, além de ampliação da pesquisa para os acadêmicos da área de saúde.
\end{abstract}

PALAVRAS-CHAVE: Ansiedade; Biomédico; Estudantes; Universidade.

\section{FACTORS ASSOCIATED WITH ANXIETY IN STUDENTS OF BIOMEDICINE}

ABSTRACT: Current study identifies factors associated with anxiety in students of the Biomedicine course. The qualitative-quantitative, descriptive and exploratory research was developed with 20 students of the $7^{\text {th }}$ Phase of the Biomedicine Course of the Universidade do Extremo Sul de Catarinense. Hamilton's Anxiety Assessment Scale and a semi-structured questionnaire were applied with regard to factors related to anxiety of students, teaching-learning process, evaluation, professional communication, interpersonal relationship, time administration, professional training and the course-conclusion monograph. Results showed $65 \%$ of students had low anxiety level; $25 \%$ mild anxiety level; $10 \%$ intense anxiety level. It is highly important that students should focus on self-care and self-knowledge. Further studies should be undertaken with a greater number of students at different phases of the Biomedicine course, with extensions to other students in the Health area.

KEY WORDS: Biomedicine; Students; University; Anxiety. 


\section{INTRODUÇÃO}

Oingressonoensinosuperioréumacontecimento significativo na vida do estudante, coincidindo com um período do desenvolvimento psicossocial marcado por mudanças importantes, como o período de explorações, a idade das possibilidades e instabilidades, sendo que $o$ acadêmico vivencia na universidade um processo de transição complexo (OSSE; COSTA, 2011).

Com a entrada na vida acadêmica podem ocorrer mudanças ambientais, como na rotina e nos sistemas de suporte social, como resultado do afastamento do jovem do ambiente familiar e da rede social anterior ao ensino superior. $\mathrm{O}$ ingresso na universidade pode gerar instabilidade no acadêmico que pode apresentar medo, preocupação, dúvida e ansiedade em relação ao processo de ensino-aprendizagem (OSSE; COSTA, 2011).

A ansiedade é responsável por preparar o indivíduo para situações de ameaça e perigo. Juntamente com o medo, eles envolvem fatores cognitivos, comportamentais, afetivos, fisiológicos e neurológicos que modulam a percepção do indivíduo ao ambiente, provocando respostas específicas e direcionando a algum tipo de ação. A ansiedade pode ser definida como uma condição orientada para o futuro, caracterizada por: apreensão relativa à percepção de não poder controlar ou prever eventos potencialmente aversivos; sintomas corporais de tensão física; e desvio do foco de atenção para esses eventos potencialmente aversivos ou às respostas afetivas eliciadas por eles (DESOUSA et al., 2013).

A ansiedade pode estar relacionada a diversos distúrbios psicológicos podendo ser considerado um transtorno mental dependendo do nível da ansiedade e de fatores associados (BORINE, 2011). "A ansiedade social é um dos problemas mais frequentes de saúde mental" (BOLSONI-SILVA; LOUREIRO, 2014, p. 223).

$O$ estresse e a ansiedade têm sido evidenciados de maneira significativa entre os acadêmicos da área da saúde. A ansiedade está presente no período de formação do profissional, pois é onde o estudante se depara com situações desafiadoras que interferem, muitas vezes, no seu processo de aprendizado e nas suas condições de saúde (COSTA; POLAK, 2009).
A ansiedade influencia negativamente na qualidade de vida dos estudantes e está intimamente ligada ao desempenho no processo de formação e na realização das atividades acadêmicas (BAMPI et al., 2013a). Depressão e ansiedade afetam os alunos tanto profissionalmente e pessoalmente, com dificuldades no relacionamento interpessoal, abuso de drogas, deterioração do desempenho acadêmico, diminuição da empatia e adoecimento físico e mental (TABALIPA et al., 2015).

Segundo o Conselho Federal de Biomedicina, o biomédico é um profissional dotado de conhecimentos $\mathrm{e}$ habilidades que lhe possibilitam comunicação, liderança, atenção à saúde, à gestão administrativa, à tomada de decisões, educação permanente (CRBM, 2009; CRBM, 2015).

A liderança é uma das habilidades que os estudantes de Biomedicina aprendem a desenvolver no decorrer da vida acadêmica (CRBM, 2015).

\begin{abstract}
Conflitos entre pessoas, escassez de materiais e imprevistos, são exemplos de situações que fazem parte da rotina de um Biomédico, exigindo céleres providências. Avaliar, sistematizar e decidir quanto ao uso apropriado de medicamentos, de equipamentos, de procedimentos, de práticas, bem como gerenciar a força de trabalho, dos recursos materiais e de informação para garantir a eficácia e eficiência dos trabalhos também são atribuições do Biomédico (CFBM, 2015, p. 2).
\end{abstract}

Assim, debater sobre o cuidado, suas formas de ser e agir, é primordial para desenvolver nos profissionais de saúde a necessidade de se autoconhecer e se reconhecer, a fim de valorizar o cuidar de si para poder cuidar do outro (SANTOS; RADUNZ, 2011). "Cuidar das pessoas pressupóe contribuir para a melhoria da sua vida e, de certo modo, é importante cuidar de si para ter condições de fazê-lo pelo outro" (BAMPI et al., 2013a, p. 131).

Como acadêmica do curso de Biomedicina constatou-se a ansiedade dos estudantes em relação à realização do Trabalho de Conclusão de Curso, além das atividades acadêmicas e estágios curriculares inerentes ao curso. A ansiedade pode ocasionar processos patológicos quando o seu grau está desmedido interferindo no 
processo de ensino-aprendizagem do acadêmico, sendo necessária a implementação de medidas de prevenção à saúde mental.

Durante o período acadêmico 15 a $29 \%$ de estudantes universitários podem apresentar transtorno de ansiedade durante sua vida acadêmica (BRANDTNER; BARDAGI, 2010). O ingresso na universidade é considerado uma experiência geradora de ansiedade aos estudantes em virtude da época de transição que o ofício de estudar representa. Desta forma é imprescindível conhecer como os estudantes enfrentam as adversidades e dificuldades na universidade e a repercussão da ansiedade na saúde mental (TEIXEIRA et al., 2008).

Nesta perspectiva este estudo teve por objetivo identificar os fatores associados à ocorrência de ansiedade dos acadêmicos da $7^{\mathrm{a}}$ fase do curso de Biomedicina.

\section{MATERIAL E MÉTODOS}

Pesquisa de abordagem quali-quantitativa, descritiva e exploratória. No segundo semestre de 2015 estavam matriculados na $7^{\mathrm{a}}$ fase do curso de Biomedicina 27 acadêmicos, sendo que aceitaram participar da pesquisa 20 acadêmicos.

$\mathrm{Na}$ fase 1 aplicou-se a Escala de Avaliação de Ansiedade de Hamilton com os acadêmicos participantes do estudo. $\mathrm{Na}$ fase 2 aplicou-se questionário semiestruturado sobre os fatores relacionados à ansiedade com acadêmicos que apresentaram nível de ansiedade leve, moderado e severo.

Dos 20 acadêmicos que responderam a Escala de Avaliação de Ansiedade de Hamilton, 16 acadêmicos (80\%) aceitaram responder o questionário semiestruturado sobre os fatores relacionados à ansiedade dos universitários.

Como critério de inclusão utilizou-se os acadêmicos da $7^{\mathrm{a}}$ fase do curso de Biomedicina que estavam realizando o TCC, que aceitaram participar da pesquisa de acordo com a Resolução 466/12 e assinaram o Termo de Consentimento Livre e Esclarecido (TCLE).

Realizou-se a análise e interpretação dos dados qualitativos através da análise de conteúdo, a partir da categorização dos dados, com a ordenação, classificação e análise final dos dados pesquisados (MINAYO, 2009). Categoria refere-se a um conceito que abrange elementos ou aspectos com características comuns ou que se relacionam entre si, são estabelecidas para classificar os eventos. Categorizar é agrupar elementos, ideias ou expressões em torno de um conceito (MINAYO, 2009).

$\mathrm{Na}$ fase de análise de dados quantitativos, as informações foram quantificadas através do Microsoft Office Excel.

A Escala de Ansiedade de Hamilton (1959) compreende 14 itens distribuídos em dois grupos, sendo o primeiro grupo com 7 itens, relacionado a sintomas de humor ansioso e o segundo grupo, também com 7 itens, relacionado a sintomas físicos de ansiedade. Segundo Bandeira (2010), dentre as escalas de humor utilizadas mundialmente, traduzidas e adaptadas para a realidade brasileira para o quesito "ansiedade", a escala de Hamilton-A para Ansiedade apresenta fácil aplicabilidade e confiabilidade (HAMILTON, 1959).

O escore total é obtido pela soma dos valores (graus) atribuídos em todos os 14 itens da escala, cujo resultado varia de 0 a 56. Os Graus de Ansiedade segundo a Escala de Ansiedade de Hamilton são: Nenhum = 0; Leve $=1 ;$ Médio $=2 ;$ Forte $=3 ;$ Máximo $=4$.

A soma dos escores obtidos em cada item resulta em um escore total, que varia de 0 a 56 . Esse escore deve ser classificado de acordo com os intervalos a seguir: 0 (zero) caracteriza ausência de ansiedade; 1 a 17 pontos ansiedade leve; 18 a 24 pontos ansiedade moderada; 25 a 56 pontos ansiedade severa ou intensa.

Para preservar o sigilo e o anonimato dos acadêmicos pesquisados, utilizou-se indicador alfanumérico, significando biomédico (B1 a B20). A pesquisa foi aprovada pelo Comitê de Ética em Pesquisa da UNESC pelo Projeto $n^{0} 1.029 .241 / 2015$.

\section{RESULTADOS}

Nos resultados da pesquisa apresentou-se o grau de ansiedade de acadêmicos de Biomedicina $(\mathrm{n}=20)$ segundo a Escala de Avaliação de Ansiedade de Hamilton e os resultados do questionário semiestruturado sobre os fatores relacionados à ansiedade dos universitários (n $=16$ ). 
3.1 ESCALA DE AVALIAÇÃO DE ANSIEDADE DE HAMILTON

Após a seleção dos acadêmicos, a partir dos critérios de inclusão da pesquisa, realizou-se o teste de ansiedade de Hamilton com 20 acadêmicos da $7^{\text {a }}$ fase do curso de Biomedicina que estavam realizando o TCC. Dos participantes do estudo $75 \%$ (12) são do sexo feminino e $25 \%$ (04) do sexo masculino, com idade variando de 20 a 45 anos. Quanto ao estado civil 87,5\% (14) são solteiros e $12,5 \%$ (02) casados.

Todos os acadêmicos apresentaram algum nível de ansiedade, sendo classificados em: 65\% nível leve; $25 \%$ nível moderado e $10 \%$ nível intenso.

\subsection{OS FATORES RELACIONADOS À OCORRÊNCIA DE ANSIEDADE NOS ACADÊMICOS DE BIOMEDICINA}

Os fatores relacionados à ocorrência de ansiedade em relação às atividades teóricas e o processo de avaliação apontam que 56,25\% dos acadêmicos sentem insegurança ou medo para realizar as provas teóricas e práticas; $43,75 \%$ relatam excesso de atividades curriculares; $18,75 \%$ questionam a forma adotada para avaliar o conteúdo teórico; $12,5 \%$ citam como agente estressor o tempo exigido pelo professor para a entrega das atividades extraclasse além da dificuldade com o conteúdo programático e as atividades desenvolvidas.

Tabela 1. Fatores Relacionados à Ocorrência de Ansiedade em Relação às Atividades Teóricas x Processo de Avaliação ( $\mathrm{n}=$ 16)

\begin{tabular}{lcc}
\hline $\begin{array}{l}\text { Atividades teóricas x processo } \\
\text { de avaliação }\end{array}$ & Número & $\begin{array}{c}\text { Porcentagem } \\
\text { (\%) }\end{array}$ \\
\hline $\begin{array}{l}\text { Sentir insegurança ou medo } \\
\text { para realizar as provas teóricas e } \\
\text { práticas }\end{array}$ & 09 & 56,25 \\
$\begin{array}{l}\text { Excesso de atividades curriculares } \\
\text { A forma adotada para avaliar o }\end{array}$ & 07 & 43,75 \\
$\begin{array}{l}\text { conteúdo teórico } \\
\begin{array}{l}\text { Tempo exigido pelo professor para } \\
\text { a entrega das atividades extraclasse }\end{array}\end{array}$ & 02 & 18,75 \\
$\begin{array}{l}\text { Dificuldade com o conteúdo } \\
\text { programático, as atividades } \\
\text { desenvolvidas e a metodologia de } \\
\text { ensino adotada }\end{array}$ & 02 & \\
\hline
\end{tabular}

Fonte: Dados da pesquisa, 2015.

Em relação à comunicação profissional e relacionamento interpessoal, $18,75 \%$ dos acadêmicos citaram falta de comunicação clara entre professor- aluno e falta de acolhimento no campo de estágio; $12,5 \%$ conflito na relação interpessoal com os colegas de turma e falta de conhecimento da equipe multiprofissional sobre a atuação do biomédico na prática profissional e $6,25 \%$ dificuldades que envolvem o relacionamento com outros profissionais da área.

Tabela 2. Fatores relacionados à ocorrência de ansiedade em relação à Comunicação Profissional e Relacionamento Interpessoal $(n=16)$

\begin{tabular}{lcc}
\hline $\begin{array}{l}\text { Comunicação Profissional e } \\
\text { Relacionamento Interpessoal }\end{array}$ & No & $\begin{array}{c}\text { Porcentagem } \\
\text { (\%) }\end{array}$ \\
\hline $\begin{array}{l}\text { Falta de comunicação clara entre } \\
\text { professor x aluno }\end{array}$ & 03 & 18,75 \\
$\begin{array}{l}\text { Falta de acolhimento no campo de } \\
\text { estágio dos acadêmicos }\end{array}$ & 03 & 18,75 \\
$\begin{array}{l}\text { Conflito na relação interpessoal } \\
\text { com os colegas de turma }\end{array}$ & 02 & 12,5 \\
$\begin{array}{l}\text { Falta de conhecimento da } \\
\text { equipe multiprofissional sobre a } \\
\text { atuação do biomédico na prática } \\
\text { profissional }\end{array}$ & 02 & 12,5 \\
$\begin{array}{l}\text { As dificuldades que envolvem } \\
\text { o relacionamento com outros } \\
\text { profissionais da área }\end{array}$ & 01 & \\
\hline
\end{tabular}

Fonte: Dados da pesquisa, 2015.

Diante do gerenciamento do tempo 56,25\% citam falta de tempo para os momentos de descanso; 50\% relatam tempo reduzido para estar com os familiares, amigos, namorado(a) ou falta de tempo para o lazer; $37,5 \%$ apresentam problemas familiares que interferem na vida acadêmica; $31,25 \%$ estão com problema de saúde/emocional que interfere na vida acadêmica e $25 \%$ afirmam problemas financeiros, com dificuldades para pagar a mensalidade. 
Tabela 3. Fatores relacionados à ocorrência de ansiedade em relação ao Gerenciamento do Tempo $(n=16)$

\begin{tabular}{lcc}
\hline Gerenciamento do Tempo & Número & $\begin{array}{c}\text { Porcentagem } \\
\text { (\%) }\end{array}$ \\
\hline $\begin{array}{l}\text { Faltar tempo para os momentos de } \\
\text { descanso }\end{array}$ & 09 & 56,25 \\
$\begin{array}{l}\text { Tempo reduzido para estar com a } \\
\text { família, amigos, namorado(a) }\end{array}$ & 08 & 50 \\
$\begin{array}{l}\text { Faltar tempo para o lazer } \\
\begin{array}{l}\text { Problemas familiares que } \\
\text { interferem na minha vida }\end{array}\end{array}$ & 08 & 50 \\
acadêmica & 06 & 37,5 \\
$\begin{array}{l}\text { Estou com problema de saúde/ } \\
\text { emocional que interfere na minha } \\
\text { vida acadêmica }\end{array}$ & 05 & 31,25 \\
$\begin{array}{l}\text { Problemas financeiros com } \\
\text { dificuldades para pagar a } \\
\text { mensalidade }\end{array}$ & & \\
\hline
\end{tabular}

Fonte: Dados da pesquisa, 2015.

Em relação aos fatores ambientais observouse que $37,5 \%$ dos acadêmicos relataram distância entre a faculdade e o local de moradia e 6,25\% moram longe da família para poder cursar a universidade, relatando saudade e ansiedade.

\section{A formação profissional apresentou um} resultado significativo, onde $75 \%$ dos acadêmicos descreveram ter preocupação com o futuro profissional; 56,25\% diante do mercado de trabalho; $43,75 \%$ pensar nas situações que poderão vivenciar quando atuar como biomédico e as novas situações que poderão vivenciar na prática clínica; $31,25 \%$ dúvidas e insegurança em relação ao perfil de liderança e empreendedorismo; $18,75 \%$ carga horária teórica x prática insuficiente para a formação com segurança do profissional; $6,25 \%$ perceber a responsabilidade profissional quando está atuando no campo de estágio e a síndrome do excesso de informação.
Tabela 4. Fatores relacionados à ocorrência de ansiedade em relação à Formação Profissional $(\mathrm{n}=16)$

\begin{tabular}{lcc}
\hline Formação Profissional & Número & $\begin{array}{c}\text { Porcentagem } \\
\text { (\%) }\end{array}$ \\
\hline $\begin{array}{l}\text { Ter preocupação com o futuro } \\
\text { profissional }\end{array}$ & 12 & 75 \\
$\begin{array}{l}\text { Campo de trabalho para o } \\
\text { biomédico/empregabilidade }\end{array}$ & 09 & 56,25 \\
$\begin{array}{l}\text { Pensar nas situações que poderá } \\
\text { vivenciar quando for biomédico/ }\end{array}$ & 07 & 43,75 \\
$\begin{array}{l}\text { As novas situações que poderá } \\
\text { vivenciar na prática clínica }\end{array}$ & & \\
$\begin{array}{l}\text { Dúvidas e insegurança em relação } \\
\text { ao meu perfil de liderança e } \\
\text { empreendedorismo }\end{array}$ & 05 & 31,25 \\
$\begin{array}{l}\text { Carga horária teórica x prática } \\
\text { insuficiente para a formação com } \\
\text { segurança do profissional }\end{array}$ & 03 & 18,75 \\
$\begin{array}{l}\text { Perceber a responsabilidade } \\
\text { profissional quando está atuando } \\
\text { no campo de estágio }\end{array}$ & & \\
$\begin{array}{l}\text { A Síndrome do Excesso de } \\
\text { Informação }\end{array}$ & 01 & 6,25 \\
\hline
\end{tabular}

Fonte: Dados da pesquisa, 2015.

Em relação aos fatores vinculados à ocorrência de ansiedade em relação ao Trabalho de Conclusão de Curso (TCC) 50\% dos acadêmicos ressaltaram as dificuldades relativas à coleta de dados do TCC e o medo de apresentar o TCC na banca examinadora; 31,25\% citaram o medo da reprovação e $25 \%$ as dificuldades relativas à análise de dados do TCC.

Tabela 5. Fatores relacionados à ocorrência de ansiedade em relação ao Trabalho de Conclusão de Curso (TCC) $(\mathrm{n}=16)$

\begin{tabular}{lcc}
\hline TCC & No & $\begin{array}{c}\text { Porcentagem } \\
\text { (\%) }\end{array}$ \\
\hline $\begin{array}{l}\text { Dificuldades relativas à coleta de dados } \\
\text { do TCC }\end{array}$ & 08 & 50 \\
$\begin{array}{l}\text { Medo de apresentar o TCC na banca } \\
\text { examinadora }\end{array}$ & 08 & 50 \\
$\begin{array}{l}\text { Medo de não passar neste semestre em } \\
\text { virtude do TCC }\end{array}$ & 05 & 31,25 \\
$\begin{array}{l}\text { Dificuldades relativas à análise de dados } \\
\text { do TCC }\end{array}$ & 04 & 25 \\
\hline $\begin{array}{l}\text { Fonte: Dados da pesquisa, 2015. } \\
\text { M }\end{array}$ &
\end{tabular}

Em relação às Estratégias de Enfrentamento 
da Ansiedade que o acadêmico poderá utilizar para melhorar a qualidade de vida, 12,5\% dos acadêmicos citaram medicamentos, acupuntura e relaxar na praia; $6,25 \%$ utilizar terapias alternativas, reiki, massagem, exercícios, dormir mais, trabalhar menos e administrar melhor seu tempo.

\section{DISCUSSÃO}

Os resultados do presente estudo evidenciaram que todos os acadêmicos de Biomedicina possuem ansiedade, sendo 65\% nível leve; $25 \%$ nível moderado e 10\% nível intenso. Estudos de Baptista (2006); Tabalipa et al. (2015); Vergara; Cárdenas; Martínez (2014); Serra; Dinato; Caseiro (2015); Bampi et al. (2013) encontraram níveis significativos de ansiedade nos acadêmicos.

Em um estudo produzido por Baptista (2006), o qual investigou o transtorno da ansiedade social (TAS) em estudantes universitários brasileiros das mais variadas áreas, como biológicas, humanas e exatas, foi observada uma prevalência de 11,6\% de transtorno nos alunos universitários, sendo considerado como um índice elevado.

Em pesquisa de Tabalipa et al. (2015) foi encontrada prevalência de ansiedade de 35,5\%, e depressão 32,8\%, em acadêmicos de Medicina, sendo superior à média encontrada na população em geral.

A prevalência de sintomas ansiosos de 973 estudantes universitários da cidade de Cartagena, na Colômbia, foi de 76,2\% (VERGARA; CÁRDENAS; MARTÍNEZ, 2014).

Em pesquisa de Serra; Dinato; Caseiro (2015), com amostra de 657 alunos de Medicina, realizada na cidade de Santos, região do litoral paulista, foi demonstrado que $21 \%$ dos estudantes apresentaram ansiedade.

Em estudo de Bampi et al. (2013a), com objetivo de conhecer a percepção sobre qualidade de vida de 84 estudantes de graduação em Medicina da Universidade de Brasília, foi possível evidenciar que o domínio psicológico foi o que recebeu o mais baixo escore na avaliação: 95,2\% dos entrevistados apresentaram sentimentos negativos (mau humor, desespero, ansiedade e depressão) e, destes, $50 \%$ experimentaram esses sentimentos frequentemente, muito frequentemente ou sempre.

Outra pesquisa de Bampi et al. (2013b) objetivou conhecer a percepção sobre a qualidade de vida com 56 acadêmicos de Enfermagem da Faculdade de Ciências da Saúde da Universidade de Brasília. As facetas denominadas capacidade de concentração, sono, grau de energia diário, capacidade para realizar atividades do dia a dia e para o trabalho, oportunidades de lazer, recursos financeiros e sentimentos negativos demonstraramse comprometidas. Essas facetas influenciaram negativamente na qualidade de vida dos estudantes, podendo desencadear sentimentos negativos, como mau humor, desespero, ansiedade e depressão.

Estas facetas estão intimamente ligadas ao sucesso no processo de aprendizagem e na realização das atividades acadêmicas e em conjunto, podem ser desencadeantes de sentimentos negativos, os quais têm influência direta no grau de satisfação/insatisfação e na qualidade de vida (BAMPI et al., 2013b).

Os fatores relacionados à ocorrência de ansiedade nos acadêmicos de Biomedicina denotam a insegurança ou medo para realização de provas teóricas e práticas; excesso de atividades curriculares; processo de avaliação do conteúdo teórico; tempo exigido pelo professor para a entrega das atividades extraclasse além da dificuldade inerente ao processo de ensino-aprendizagem.

A pesquisa de Serra; Dinato; Caseiro (2015) evidenciou alta prevalência de sintomas depressivos e ansiedadeemestudantesdeMedicinasendopossivelmente relacionada com fatores estressantes inerentes à formação acadêmica, associada com características psicodinâmicas. Alguns dos alunos, como consequência de sua própria personalidade, demonstram aflição emocional mais aguda e maiores dificuldades para enfrentar questões conflitantes, tornando-se mais vulneráveis a distúrbios emocionais e, consequentemente, a situações de risco.

Kaplan; Sadock; Grebb (2006); Holmes (1997) relatam que a ansiedade afeta a cognição, alterando o aprendizado, produzindo confusão e distorções perceptivas, não apenas em termos de tempo e espaço, mas de pessoas e de significados dos eventos. Essas distorções podem interferir no aprendizado, baixando a concentração, reduzindo a memória e prejudicando a capacidade de associação, podendo também ser 
observado nos resultados desta pesquisa.

Em relação ao gerenciamento do tempo os acadêmicos citaram a falta de tempo para os momentos de descanso; tempo reduzido para estar com a família, amigos, namorada(o); e falta de tempo para o lazer o que possibilita a ocorrência de ansiedade.

Pesquisa de Bampi et al. (2013b) apontou a insatisfação dos estudantes de Enfermagem com a falta de tempo para atividades de lazer podendo estar diretamente relacionada aos problemas com sono e falta de energia. O estudo de Bampi et al. (2013a) também evidenciou a insatisfação dos futuros médicos com a falta de tempo para atividades de lazer tendo estreita relação com a carga horária exigida e o período de sua juventude.

Os acadêmicos também relataram problemas familiares e de saúde, além de problemas financeiros que interferem na vida acadêmica e podem ocasionar ansiedade.

As dificuldades financeiras foram apontadas como fator que afeta diretamente a qualidade dos acadêmicos de Enfermagem, em estudo de Bampi et al. (2013b). Os fatores associados com sintomas ansiosos podem estar relacionados às dificuldades financeiras; problemas familiares; e consumo de álcool. Devem ocorrer intervenções que possibilitem a qualidade de vida para os universitários (VERGARA; CÁRDENAS; MARTÍNEZ, 2014).

Os fatores ambientais também foram destacados nesta pesquisa como fator causal da ansiedade em virtude da distância entre a Universidade e o local de moradia e morar longe da família. Com o surgimento da necessidade de deixar a casa dos pais e começar uma vida longe das facilidades do lar, cria-se liberdade, sendo que a ansiedade acaba se tornando parte inevitável desse processo. Há casos mais extremos de saudade que podem gerar sintomas como crises de choro, aperto no peito e desconforto em qualquer tipo de ambiente (MACHADO, 2013).

A preocupação com a formação e o futuro profissional foi destacada como fator relacionado à ansiedade dos acadêmicos de Biomedicina. Uma das causas para a alta taxa de sintomas depressivos e ansiedade nos estudantes de Medicina está relacionada à preocupação com o futuro (TABALIPA et al., 2015).

O biomédico é um profissional da área da saúde com formação generalista, humanista, crítica e reflexiva, capacitado a atuar em todos os níveis do sistema de saúde. O conteúdo programático do curso de Biomedicina objetiva dotar o aluno de conhecimentos e habilidades que lhe possibilitam comunicação, liderança, atenção à saúde, à gestão administrativa, à tomada de decisões, educação permanente (CRBM, 2009; CRBM, 2015).

A grade curricular deve estar relacionada a todo o processo saúde/doença do cidadão, da família e da comunidade, integrada à realidade epidemiológica e profissional (CRBM, 2009; CRBM, 2015). O conteúdo contempla: ciências exatas; ciências biológicas; ciências humanas e sociais; e ciências da Biomedicina; incluemse os conteúdos teóricos e práticos relacionados com a saúde, doença e meio ambiente, com ênfase nas áreas de citopatologia, genética, biologia molecular, ecoepidemiologia das condições de saúde e dos fatores predisponentes à doença e serviços complementares de diagnóstico laboratorial em todas as áreas da Biomedicina (CRBM, 2009; CRBM, 2015).

A carga horária do curso é definida pelo Ministério da Educação, por meio da Resolução ${ }^{0}$ 4, de 06 de abril de 2009, da Câmara de Educação Superior do Conselho Nacional de Educação, que estipula a carga horária mínima de 3.200 horas/relógio (60 minutos). Porém, a recomendação contida na Resolução $n^{0} 126$, de 16 de junho de 2006 do CFBM, é para que as Escolas mantenham seus cursos com carga horária mínima de 4.000 horas-aula (50 minutos), priorizando sua parte prática com 600 horas-aula, no mínimo, e 500 horas-aula para cada habilitação implantada (CRBM, 2009; CRBM, 2015).

De acordo com o Conselho Federal de Biomedicina os procedimentos técnico-operacionais executados pelos biomédicos podem ser agrupados em 3 grandes áreas de atuação, obedecida a habilitação necessária: Diagnóstico; Coordenação, Direção, Chefia, Perícia, Auditoria, Supervisão e Ensino; Pesquisa e Investigação (CRBM, 2009; CRBM, 2015).

"Há no Brasil, hoje, mais de 30.000 (trinta mil) biomédicos em atividade" (CRBM, 2015, p. 3). "O profissional biomédico atua em pesquisas que podem fornecer dados desde o diagnóstico até a descoberta científica para a cura e prevenção de doenças que ainda 
devastam a população em geral" (SILVA et al., 2014, p. 1).

O biomédico é capacitado a desenvolver métodos para a promoção da saúde e prevenção de doenças, bem como pesquisar novas técnicas a serviço do bemestar social. O biomédico tem de tomar várias decisões importantes e decisivas diariamente, como, por exemplo: decidir quanto ao uso apropriado de medicamentos, equipamentos, procedimentos e de práticas. Por isso é de extrema importância que tal profissional detenha a capacidade de iniciativa e comunicação (CRBM, 2015).

Embora seja ampla a área de atuação do biomédico, a de Análises Clínicas ainda é a mais procurada pelos profissionais do setor (GUIA DO ESTUDANTE, 2015; CRBM, 2015). "No Brasil, cerca de $80 \%$ dos Biomédicos trabalham no segmento. O mercado do diagnóstico laboratorial é gigantesco" (CRBM, 2015, p. 11).

Segundo o Guia do Estudante (2015) pequena parte dos biomédicos segue carreira acadêmica, lecionando ou fazendo pesquisa. Mas o bacharel pode atuar em órgãos públicos de saúde e em indústrias de biotecnologia, em áreas como análise de alimentos. Boas perspectivas têm surgido nas áreas de citopatologia, toxicologia e reprodução humana. Aárea de criminalística é uma promessa de crescimento do mercado.

A área de atuação do Biomédico é ampla e o profissional pode se formar em várias habilitações, todas regulamentadas pelo Conselho Federal de Biomedicina - CFBM. Pesquisadores brasileiros da área de Biomedicina têm se destacado em estudos de repercussão mundial, como o Projeto Genoma Humano. Existem em todo o país cerca de 5.000 (cinco mil) laboratórios de análises clínicas e citologia, cuja responsabilidade técnica é exercida por biomédicos. Há no Brasil, hoje, mais de 30.000 (trinta mil) biomédicos em atividade (CRBM, 2015, p. 5).

Desta forma, considera-se que o campo de trabalho para o biomédico é promissor, em virtude das necessidades emergentes na área de biotecnologia associadas às novas pesquisas na área da saúde.

A Síndrome do Excesso de Informação foi relacionada aos fatores associados à ansiedade pelos acadêmicos. O excesso de informação provoca uma angústia típica dos tempos atuais, sendo que saber demais pode ser um problema. A geração do século XXI sofre as consequências desta síndrome. Quanto mais informações obtêm, mais ficam sabendo da existência de novas fontes da mesma informação, gerando ainda mais ansiedade (BRAGA, 2015).

Os acadêmicos de Biomedicina também vincularam a ocorrência de ansiedade em virtude da elaboração do Trabalho de Conclusão de Curso (TCC).

$\mathrm{O}$ processo de preparação que antecede o Trabalho de Conclusão de Curso é um período de muitas incertezas e inseguranças para o pré-formando. Esse contexto contribui para o surgimento da ansiedade que pode interferir no cotidiano do estudante. A sensação de despreparo, de obrigação de ter realizado um trabalho bom e o fato de ser uma decisão definitiva para sua vida faz com que os pré-formando se sintam mais ansiosos. Desta forma, esse período de preparo para o futuro biomédico acaba tornando-se um agente estressor para estes alunos (D'AVILA; SOARES, 2006).

As estratégias de enfrentamento da ansiedade que o acadêmico utiliza para melhorar a qualidade de vida estão relacionadas ao tratamento medicamentoso, acupuntura e relaxar na praia; utilizar terapias alternativas, reiki, massagem, exercícios, dormir mais, trabalhar menos e administrar melhor seu tempo.

As terapêuticas holísticas incitam mudanças em hábitos de vida e estimulam a participação ativa do sujeito, objetivando a qualidade de vida e o viver em equilíbrio através do autoconhecimento (NEVES; SELLI; JUNGES, 2010).

O reiki é uma prática milenar de imposição de mãos (Reiki), e consiste na prática de harmonização do campo energético humano (CEH) através do uso consciente das mãos, com o intuito de restabelecer pessoas enfermas (GOMES; SILVA; ARAUJO, 2008). O reiki ajuda no relaxamento físico-mental e melhora da função imunológica (PRIMO; AMORIM; LEITE, 2011).

A energia chamada reiki é um método que se adapta aos novos paradigmas da saúde, que emerge na área de saúde e inclui consciência, corpo, mente e principalmente a prevenção. Ele contribui para $o$ equilíbrio das necessidades física, mental, emocional 
e espiritual levando a uma relação harmoniosa entre o homem, seu meio, além de autoconhecimento (SALOME, 2009).

A massagem como recurso terapêutico vem sendo reconhecida como uma das terapias mais eficazes para alívio de dores e prevenção de doenças. Tem como proposta de trabalho, dentro de uma visão holística, tornar o indivíduo consciente do seu corpo, das suas tensões, da sua respiração e das suas cargas emocionais, bem como melhorar a nutrição dos tecidos pelo aumento da circulação sanguínea e linfática além de outros benefícios físicos e emocionais. A terapia através da massagem pode auxiliar na eliminação ou amenização de diversos estados mentais, como depressão, ansiedade, angústia e insônia (SEUBERT; VERONESE, 2008).

A acupuntura é uma tecnologia de intervenção em saúde que aborda de modo integral e dinâmico o processo saúde-doença no ser humano. A estimulação de pontos de acupuntura provoca a "liberação, no sistema nervoso central, de neurotransmissores e outras substâncias responsáveis pelas respostas de promoção de analgesia, restauração de funções orgânicas e modulação imunitária" (BRASIL, 2013; GENIOLE; KODJAOGLANIAN; VIEIRA, 2011, p. 2).

Entre os tratamentos indicados para a ansiedade, a acupuntura, técnica da Medicina Tradicional Chinesa, é uma terapia complementar que atua diretamente nos níveis psicológico, comportamental e biológico do indivíduo. Além de aliviar a ansiedade, a acupuntura gera bem-estar e relaxamento mental, proporcionando à pessoa uma melhor qualidade de vida (CRUZ, 2012).

As Práticas Integrativas e Complementares (PICs) são práticas que estimulam os mecanismos naturais de prevenção de agravos e recuperação de saúde e contribuem para a promoção da saúde, inserção social, redução de consumo de medicamentos, aumento da autoestima e melhoria da qualidade de vida (BRASIL, 2013; GENIOLE; KODJAOGLANIAN; VIEIRA, 2011).

A sensibilização dos acadêmicos com foco no cuidado do cuidador - autocuidado - pode contribuir na humanização da assistência e no reescalonamento de valores relacionados à ética do cuidar - de si mesmo e do outro.

O curso de Biomedicina já integra em uma disciplina optativa a acupuntura, sendo incluídos conceitos relativos à Medicina Tradicional Chinesa e a auriculoterapia. A Portaria $\mathrm{n}^{0}$ 971, de 03 de maio de 2006, aprovou a Política Nacional de Práticas Integrativas e Complementares (PNPIC) no Sistema Único de Saúde (SUS), tendo como um dos objetivos incorporar e implementar as Práticas Integrativas e Complementares, na perspectiva da prevenção de agravos e da promoção e recuperação da saúde, voltada para o cuidado continuado, humanizado e integral em saúde, estimulando alternativas inovadoras e socialmente contributivas ao desenvolvimento sustentável de comunidades (BRASIL, 2013; GENIOLE; KODJAOGLANIAN; VIEIRA, 2011).

O estudo acerca da qualidade de vida dos profissionais de saúde pode possibilitar a reflexão sobre a importância do cuidado do cuidador. A responsabilidade institucional também deve se fazer presente na busca da qualidade de vida dos acadêmicos universitários (BAMPI et al., 2013a). A melhoria da qualidade de vida dos estudantes pode ter influência positiva no processo de humanização da assistência, pois o bem-estar do profissional/estudante se reflete em sua forma de cuidar do outro (BAMPI et al., 2013b).

Os acadêmicos necessitam de suporte para o enfrentamento das diversas situações que interferem em sua qualidade de vida, especialmente naquelas que estão vinculadas ao processo de formação (BAMPI et al., 2013b).

Desta forma, considera-se necessária a implementação de estratégias que permitam identificar as dificuldades vivenciadas pelos acadêmicos favorecendo a busca por soluções para os conflitos que incidem na qualidade de vida dos estudantes (BAMPI et al., 2013b).

\section{CONSIDERAÇÕES FINAIS}

Em relação ao nível de ansiedade os acadêmicos de Biomedicina apresentaram 65\% grau leve; $25 \%$ grau moderado; $10 \%$ grau intenso. Nessa perspectiva considera-se imprescindível o cuidado aos acadêmicos da Universidade, com foco no autocuidado e autoconhecimento. Cabe salientar uma inquietação: de que forma a Universidade e o curso de Biomedicina 
poderão alicerçar uma melhoria da qualidade de vida do acadêmico, possibilitando uma melhor aprendizagem e formação de vínculos terapêuticos, onde com isto possibilitaria a melhoria da realidade dos acadêmicos, perante o penúltimo semestre do curso de Biomedicina, com a realização do TCC.

Sugere-se desta forma um aprofundamento da presente pesquisa, com maior número de acadêmicos, contemplando as diversas fases do curso, além de ampliação da pesquisa para os acadêmicos da área de saúde em geral.

\section{REFERÊNCIAS}

BAMPI, L. N. S.; BARALDI, S.; GUILHEM, D.; ARAÚJO, M. P.; CAMPOS, A. C. O. Qualidade de vida de estudantes de medicina da Universidade de Brasília. Rev. bras. educ. med., Rio de Janeiro, v. 37, n. 2, p. 217-225, jun. 2013. Disponível em: <http://www. scielo.br/scielo.php?script $=$ sci_arttext\&pid $=S 0100$ 55022013000200009\&lng $=$ pt\&nrm $=$ iso $>$. Acesso em: 07 jul. 2016.

BAMPI, L. N. S.; BARALDI, S.; GUILHEM, D.; POMPEU, R. B.; CAMPOS, A. C. O. Percepção sobre qualidade de vida de estudantes de graduação em enfermagem. Rev. Gaúcha Enferm., Porto Alegre, v. 34, n. 2, p. 125-132, jun. 2013. Disponível em: <http://www. scielo.br/scielo.php?script $=$ sci_arttext\&pid $=$ S1983$14472013000200016 \& \operatorname{lng}=$ en\&nrm $=$ iso $>$. Acesso em: 07 jul. 2016. http://dx.doi.org/10.1590/S198314472013000200016 .

BANDEIRA, R. A. Dor pós-operatória em idosos submetidos à prostatectomia transvesical: correlação com a ansiedade no pré-operatório. 2010. 83f. Dissertação (Mestrado em Gerontologia) - Universidade Católica de Brasília, Brasília, 2010.

\section{BAPTISTA, C. A. Estudo da prevalência do transtorno de} ansiedade social em estudantes universitários. 2006. Dissertação (Mestrado em Saúde Mental) - Universidade de São Paulo, Ribeirão Preto, 2006. Disponível em: $<$ https://psicologado.com/psicopatologia/transtornospsiquicos/fobia-social-a-incidencia-em-estudantesuniversitarios > . Acesso em: 03 jun. 2015.
BOLSONI-SILVA, A. T.; LOUREIRO, S. R. The Role of Social Skills in Social Anxiety of University Students. Paidéia (Ribeirão Preto), Ribeirão Preto, v. 24, n. 58, p. 223232, ago. 2014. Disponível em: <http://www.scielo.br/ scielo.php?script $=$ sci_arttext\&pid $=$ S0103-863X201400 $0200223 \& \operatorname{lng}=$ en\&nrm $=$ iso $>$. Acesso em: 07 jul. 2016. http://dx.doi.org/10.1590/1982-43272458201410.

BORINE, M. S. Ansiedade, neuroticismo e suporte familiar: evidência de validade do Inventário de Ansiedade Traço-Estado (IDATE). 2011. 123f. Tese (Doutorado) - Programa de Pós-Graduação em Psicologia, Universidade São Francisco, Itatiba. SP.

BRAGA, R. O excesso de informação: a neurose do século XXI, 2015. Disponível em: $<$ http://www.mettodo. com.br/pdf/O\%20Excesso\%20de\%20Informacao.pdf $>$. Acesso em: 22 maio 2015.

BRANDTNER, M.; BARDAGI, M. Sintomatologia de Depressão e Ansiedade em Estudantes de uma Universidade Privada do Rio Grande do Sul. Gerais: Rev Interinstit Psicol., v. 2, n. 2, p. 81-91, 2010.

BRASIL. Conselho Nacional de Saúde. Resolução no 466, de 12 de dezembro de 2012. Disponível em: <http:// conselho.saude.gov.br/resolucoes/2012/Reso466.pdf $>$. Acesso em: 01 dez. 2014.

BRASIL. Ministério da Saúde. Secretaria de Atenção à Saúde. Departamento de Atenção Básica. Política nacional de práticas integrativas e complementares no SUS: atitude de ampliação de acesso. 2. ed. Brasília: Ministério da Saúde, 2013. 96p.

COSTA, A. L. S.; POLAK, C. Construção e validação de instrumento para Avaliação de Estresse em Estudantes de Enfermagem (AEEE). Rev. esc. enferm. USP. São Paulo, v. 43, n. spe, dez, 2009. Disponível em: <http://www. scielo.br/scielo.php?script $=$ sci_arttext $\&$ pid $=S 0080$ $62342009000500005 \& \operatorname{lng}=\mathrm{en \& nrm}=$ iso $>$ Acesso em: 25 mar. 2015.

CRBM. Conselho Regional de Biomedicina. Biomedicina: um painel sobre o profissional e a profissão. 2009. Disponível em: <http://www.crbm2.com.br/arquivos/ livrocrbm.pdf>. Acesso em: 15 mar. 2015. 
CRBM. Conselho Regional de Biomedicina. Manual do biomédico. 2015. Disponível em: < http://crbm1.gov.br/ MANUAL_BIOMEDICO.pdf $>$. Acesso em: 15 mar. 2015.

CRUZ, R. A. S. Como amenizar ansiedade com a acupuntura. Instituto Brasileiro de Acupuntura, 2012. Disponível em: < http://www.ibramrp.com.br/noticia/2/ como-amenizar-ansiedade-com-a-acupuntura $>$. Acesso em: 07 maio 2015.

D'AVILA, G. T.; SOARES, D. H. P. Vestibular: fatores geradores de ansiedade na 'cena da prova'. Rev bras orientac prof., v. 4, n.1/2, p. 105-16, 2006.

DESOUSA, D. A.; MORENO, A. L.; GAUER, G.; MANFRO, G. G.; KOLLER, S. H. Revisão sistemática de instrumentos para avaliação de ansiedade na população brasileira. Aval. psicol., Itatiba, v. 12, n. 3, dez, 2013. Disponível em: $\quad<$ http://pepsic.bvsalud.org/scielo.php?script $=$ sci arttext\&pid $=$ S1677-04712013000300015\&lng $=$ pt\&nrm $=$ iso $>$. Acesso em: 16 nov. 2014.

GENIOLE, L. A. I.; KODJAOGLANIAN, V. L.; VIEIRA, C. C. A. (Org.). Política Nacional de práticas integrativas e complementares no SUS. Campo Grande: Ed. da UFMS: Fiocruz Unidade Cerrado Pantanal, 2011.

GOMES, V. M.; SILVA, M. J. P.; ARAUJO, E. A. C. Efeitos gradativos do toque terapêutico na redução da ansiedade de estudantes universitários. Rev. bras. enferm. Brasília, v. 61, n. 6, dez, 2008. Disponível em: <http://www. scielo.br/scielo.php?script $=$ sci_arttext\&pid $=S 0034$ $71672008000600008 \& \operatorname{lng}=\mathrm{pt} \& \mathrm{nrm}=\mathrm{iso}>$. Acesso em: 16 fev. 2014.

GUIA DO ESTUDANTE. Biomedicina, 2015. Disponível em: <http:/guiadoestudante.abril.com.br/profissoes/ saude/biomedicina-684526.shtml > . Acesso em: 16 maio 2015.

HAMILTON, M. The assesment of anxiety states by rating. BrJ Med. Psych.1959.

HOLMES, D. S. Psicologia dos transtornos mentais. 2. ed. Porto Alegre: Artes Médicas, 1997.

KAPLAN, H. I.; SADOCK, B. J.; GREBB, J. A. Compêndio de psiquiatria: ciências do comportamento e psiquiatria clínica. 7. ed. São Paulo: Artmed, 2006.
MACHADO, R. Entenda o processo psicológico de morar longe de casa. 2013. Disponível em: < http:// www.universitario.com.br/noticias/n.php?i=14891>. Acesso em: 22 maio 2015.

MINAYO, M. C. S. Pesquisa social: teoria, método e criatividade. 28. ed. Petrópolis: Vozes, 2009. 108p.

NEVES, L. C. P.; SELLI, L.; JUNGES, R. A integralidade na Terapia Floral e a viabilidade de sua inserção no Sistema Único de Saúde. O Mundo da Saúde. São Paulo, v. 34, n. 1, p. 57-64, 2010.

OSSE, C. M. C.; COSTA, I. I. Saúde mental e qualidade de vida na moradia estudantil da Universidade de Brasília. Estud. psicol. Campinas, v. 28, n. 1, mar. 2011. Disponível em: <http://www.scielo.br/scielo. php?script $=$ sci_arttext\&pid $=$ S0103-166X20110001000 $12 \& \operatorname{lng}=$ en\&nrm $=$ iso $>$. Acesso em: 24 mar. 2015.

PRIMO, C. C.; AMORIM, M. H. C.; LEITE, F. M. C. A intervenção de enfermagem: relaxamento e seus efeitos no sistema imunológico de puérperas. Acta paul. enferm. São Paulo, v. 24, n. 6, 2011. Disponível em: < http://www. scielo.br/scielo.php?script $=$ sci_arttext $\&$ pid $=$ S0103$21002011000600003 \& \operatorname{lng}=$ en $\& n r m=i s o>$. Acesso em: 16 fev. 2014.

SALOMÉ, G. M. Sentimentos vivenciados pelos profissionais de enfermagem que atuam em Unidade Terapia Intensiva após aplicação do Reiki. Saúde Coletiva, v. 6, n. 28, p. 54-58, 2009.

SANTOS, V. E. P.; RADUNZ, V. O cuidar de si na visão de acadêmicas de enfermagem. Rev. enferm. UERJ. Rio de Janeiro, v. 19, n. 1, p. 46-51, jan./mar. 2011.

SERRA, R. D.; DINATO, S. L. M.; CASEIRO, M. M. Prevalence of depressive and anxiety symptoms in medical students in the city of Santos. J. bras. psiquiatr., Rio de Janeiro, v. 64, n. 3, p. 213-220, set. 2015. Disponível em: $\quad<$ http://www.scielo.br/scielo.php?script $=$ sci arttext\&pid $=$ S0047-20852015000300213\&lng $=$ en $\& n r m=$ iso $>$ Acesso em: 07 jul. 2016. http://dx.doi. org/10.1590/0047-2085000000081.

SEUBERT, F.; VERONESE, L. A massagem terapêutica auxiliando na prevenção e tratamento das doenças físicas e 
psicológicas. In: ENCONTRO PARANAENSE, CONGRESSO BRASILEIRO, CONVENÇÃO BRASIL/LATINO-AMÉRICA, 13., 8.,, 2., 2008. Anais... Curitiba: Centro Reichiano, 2008. Disponível em: http://www.centroreichiano.com. br/artigos/Anais\%202008/Fabiano\%20e\%20Liane.pdf Acesso em: 04 ago. 2013.

SILVA, A. R.; NUNES, C. R. S.; ARAÚJO, S. S.; VERAS, H. N. H. O papel do biomédico na saúde pública. Rev Interfaces, v. 2, n. 4, jun. 2014.

TABALIPA, F. O.; SOUZA, M. F.; PFÜTZENREUTER, G.; LIMA, V. C.; TRAEBERT, E.; TRAEBERT, J. Prevalence of Anxiety and Depression among Medical Students. Rev. bras. educ. med., Rio de Janeiro, v. 39, n. 3, p. 388-394, set. 2015. Disponível em: <http://www. scielo.br/scielo.php?script $=$ sci_arttext\&pid $=S 0100$ 55022015000300388\&lng $=$ pt\&nrm $=$ iso $>$. Acesso em: 07 jul. 2016. http://dx.doi.org/10.1590/198152712015v39n3e02662014.

TEIXEIRA, M. A. P.; DIAS, A. C. G.; WOTTRICH, S. H.; OLIVEIRA, A. M. Adaptação à universidade em jovens calouros. Psicol. Esc. Educ. (Impr.), Campinas, v. 12, n. 1, jun., 2008. Disponível em: <http://www. scielo.br/scielo.php?script $=$ sci_arttext\&pid $=$ S1413$85572008000100013 \& \operatorname{lng}=$ en\&nrm $=$ iso $>$. Acesso em: 24 mar. 2015.

VERGARA, K. M. A.; CÁRDENAS, S. D.; MARTÍNEZ, F. G. Síntomas de depresión y ansiedad en jóvenes universitarios: prevalencia y factores relacionados. Rev Clín Med Fam, v. 7, n. 1, p. 14-22, 2014.

Recebido em: 15 de janeiro de 2016 Versão final recebida em: 08 de julbo de 2016 Aceito em: 19 de julbo de 2016 